\title{
A New Decorrelation Phase Covariance Model for Noise Reduction in Unwrapped Interferometric Phase Stacks
}

\author{
Yujie Zheng, Student Member, IEEE, Howard Zebker, Fellow, IEEE, and Roger \\ Michaelides, Student Member, IEEE
}

\begin{abstract}
The accuracy of geophysical parameter estimation made with Interferometric Synthetic Aperture Radar (InSAR) time-series techniques can be improved with rapidly increasing available data volumes, and with the development of noise covariance matrices applicable to joint analysis of networks of interferograms. Here we present a new decorrelation phase covariance model and discuss its role in noise reduction in unwrapped interferometric phase stacks. We demonstrate with an example wherein we average unwrapped interferogram phase stacks that span over a transient event how a noise covariance model can aid in noise reduction. Our model suggests that, for rapidly decorrelating surfaces (i.e., surfaces with much shorter correlation time than SAR acquisition intervals), it is preferable to incorporate all available interferograms from long observation windows. For slowly decorrelating surfaces (i.e., surfaces with longer correlation time than SAR acquisition intervals), our model suggests that a small subset of interferometric pairs is sufficient. We validate our model and three existing models of decorrelation phase covariance matrices in both Cascadia - a region with heavy vegetation cover, and Death Valley - a desert region, with C-band Sentinel-1 A observations. Our proposed model matches observations with the smallest average discrepancy between theory and observations.
\end{abstract}

Index Terms-Decorrelation noise, Covariance matrix, InSAR noise reduction.

\section{INTRODUCTION}

I NTERFEROMETRIC Synthetic (InSAR) is a widely used

Aperture Radar technique that combines coherent radar images to form interferograms, which can be used to generate high-precision measurements of surface topography or crustal deformation over large areas with meter-level resolution [1]-[4]. In the past few years, three new InSAR satellites - Sentinel-1A/B and ALOS-2, became

Y. Zheng was with Stanford University, now with California Institute of Technology.

H. Zebker and R. Michaelides are with Stanford University. operational. In the near future, additional InSAR satellites such as NISAR and ALOS-4 are anticipated to come online. Featuring higher temporal sampling rates and wider spatial coverage, this new generation of satellites provides large volumes of high-quality radar measurements and thereby makes observations of mm-level signals such as interseismic velocities possible [5].

The accuracy of measured geophysical signals with InSAR is inherently limited by atmospheric noise and decorrelation. Atmospheric noise is caused by fluctuations in wave propagation delays through the atmosphere due to the presence of water vapor. Since the revisit time of SAR satellites is on the order of days, atmospheric noise is essentially uncorrelated in time but correlated in space and is often modeled as a long-wavelength artifact in individual interferograms [6]-[9]. Decorrelation, on the other hand, can be related to changes between radar measurements in surface scattering properties, imaging geometries and thermal noise among others [10], [11]. Temporal decorrelation due to independent motions of scatterers in the resolution cell translates to stochastic noise in interferometric measurements. In contrast, processes that result in both correlation loss and systematic phase shifts (e.g., variations in soil moisture [12]) have non-stochastic effects on interferometric stacks [13]-[15], and are linked with observations of phase biases in short temporal-span interferograms [16]. In this paper, we limit our focus on the first category of decorrelation that results in stochastic noise in interferometric measurements.

InSAR time-series techniques are methodologies that exploit interferogram stacks with the aim of retrieving desired geophysical signals whilst minimizing effect of decorrelation. There are two broad categories of InSAR time-series algorithms. One involves identifying "persistent scatterer" (PS) pixels with highly stable 
scattering mechanisms [17], [18]. The PS pixels are minimally affected by decorrelation but are mostly limited to man-made structures and cities. The other approach involves exploitation of pixels that are affected by decorrelation, known as "distributed scatterers" (DS) pixels. The DS method can be further divided into two categories. The first category of DS methods is based on analysis of unwrapped interferometric phase stacks. For example, the conventional Small BAseline Subset (SBAS) technique [19] obtains phase time-series by solving a linear system of equations of unwrapped phases. The SBAS method limits the effect of decorrelation by restricting use of interferometric measurements to a subset of interferograms with small spatial and temporal baselines. The second category of DS methods performs time-series estimation based on analysis of SAR correlation matrices before phase unwrapping. Accounting for target statistics of SAR measurements [20], [21], approaches such as SqueeSAR [22] and its ensuing algorithms [23]-[25] relax the coherence constraint imposed in SBAS and allow for use of all interferograms [20], [21]. In this paper, we focus on the first category of DS methods that operates on networks of unwrapped interferometric phases. Hereinafter we refer to the first category of DS methods as "SBAS-like time-series algorithms".

Phase statistics of multi-interferometric measurements play a critical part in improving the performance and design of SBAS-like time-series algorithms. Several studies have evaluated covariance between interferometric phases. In the simple mathematical framework developed in [6] to describe common noise sources in InSAR, decorrelation phase is modeled as an independent noise term, uncorrelated between interferograms. More recent work such as [13], [14], [20], [26]-[28] show that interferometric phases are partially correlated between interferograms and present analytical models with various forms. With respect to these works, we propose a new covariance model for decorrelation phase based on surface scattering characteristics. We compare and validate our model with previously published models against Sentinel-1 data collected over both low coherence and mediumto-high coherence areas. We also demonstrate with an example how a network noise covariance model can facilitate decorrelation noise reduction.

This paper is organized as follows. In section II] we review existing decorrelation phase covariance models and then present the proposed model. In section III. we demonstrate how decorrelation covariance models can be incorporated into SBAS-like time-series algorithms to great effect and provide theoretical comparisons between the existing and the proposed models. In section IV, we validate all models using data collected by Sentinel-1. We conclude with a summary in section $\mathrm{V}$

\section{Decorrelation Phase Covariance Models}

Similar to the definition of the correlation coefficient $\rho$ between radar measurements, we define $\gamma$ as the correlation coefficient between interferometric measurements. Let $\sigma_{x, i j}^{2}$ denote the variance associated with $\phi_{x, i j}^{\text {decor }}$ (by convention, $i<j$ ), the decorrelation phase component of the interferometric measurement between SAR acquisitions with indices $i$ and $j$ for pixel $\mathrm{x}$. The covariance of $\phi_{x, i j}^{\text {decor }}$ and $\phi_{x, k l}^{\text {decor }}$ is

$$
\operatorname{cov}\left(\phi_{x, i j}^{\text {decor }}, \phi_{x, k l}^{\text {decor }}\right)=\gamma\left(\phi_{x, i j}^{\text {decor }}, \phi_{x, k l}^{\text {decor }}\right) \cdot \sigma_{x, i j} \sigma_{x, k l}
$$

Phase variance $\sigma_{x, i j}^{2}$ have been comprehensively studied in literature and can either be derived from a probability distribution function (PDF) of interferometric phases under the assumption of a distributed scatterer mechanism [2], [29]-[32] or be approximated by the Cramer-Rao bound [33] when the correlation coefficient $\rho$ between radar measurements is close to 1 . The main focus of this paper is therefore the derivation of the correlation coefficient between interferometric phases $\gamma\left(\phi_{x, i j}^{\text {decor }}, \phi_{x, k l}^{\text {decor }}\right)$. In this section, we first review the existing covariance models. Then we introduce the proposed new covariance model.

It is worth emphasizing that the focus of this paper is the covariance models for multi-interferometric phase measurements of a single pixel. Hereinafter we omit the subscript $x$ to simplify mathematical notations.

\section{A. Existing Models for $\gamma\left(\phi_{i j}^{\text {decor }}, \phi_{k l}^{\text {decor }}\right)$}

The first statistical evaluation of temporal decorrelation noise is given by [6], which models decorrelation as a fully independent noise term in each interferogram in a network:

$$
\gamma\left(\phi_{i j}^{\text {decor }}, \phi_{k l}^{\text {decor }}\right)=\delta_{i k} \delta_{j l}
$$

where $\delta_{i j}=1$, if $i=j$, and $\delta_{i j}=0$ if otherwise.

In contrast, more recent work such as [13], [14], [26][28] argue that decorrelation noise is correlated between interferograms. [26] and [13] provide estimations under the simplified assumption that interferometric measurements can be described as circular complex Gaussian random variables. [28] later notes that interferometric 
phase generally does not follow a circular Gaussian distribution and provides a closed-form analytical approximation of the correlation between interferometric decorrelation phases using the method of nonlinear error propagation:

$$
\operatorname{cov}\left(\phi_{i j}^{\text {decor }}, \phi_{k l}^{\text {decor }}\right) \approx \frac{\rho_{i k} \rho_{j l}-\rho_{i l} \rho_{j k}}{2 L \rho_{i j} \rho_{k l}}
$$

where $L$ is the number of looks. Note that when $i=$ $k, j=l, 3$, becomes the Cramer-Rao bound [33], which is the lower bound for decorrelation phase variance. [14] also presents a similar equation with (3). We can derive $\gamma\left(\phi_{i j}^{\text {decor }}, \phi_{k l}^{\text {decor }}\right)$ from 3 :

$$
\gamma\left(\phi_{i j}^{\text {decor }}, \phi_{k l}^{\text {decor }}\right)=\frac{\rho_{i k} \rho_{j l}-\rho_{i l} \rho_{j k}}{\sqrt{\left(1-\rho_{i j}^{2}\right)\left(1-\rho_{k l}^{2}\right)}}
$$

Since (4) has been derived with an approximation that only holds for a large coherence, (4) is not a complete covariance model for decorrelation phase noise.

Attempting to extend the covariance model from high to moderate coherence level, [27] starts with a pseudocovariance matrix $\tilde{\Omega}_{i f g}$ that is derived from a SAR coherence matrix $\Omega_{\text {sar }}$ and the InSAR incidence matrix $A$ (Definition of $A$ can be found in [19]): $\tilde{\Omega}_{i f g}=$ $\frac{1}{2} A \Omega_{\text {sar }} A^{T}$. The pseudocovariance of $\phi_{i j}^{\text {decor }}$ and $\phi_{k l}^{\text {decor }}$ is then expressed as

$$
\widetilde{\operatorname{cov}}\left(\phi_{i j}^{\text {decor }}, \phi_{k l}^{\text {decor }}\right)=\frac{\rho_{i k}+\rho_{j l}-\rho_{i l}-\rho_{j k}}{2}
$$

where $\rho_{i j}$ is the correlation coefficient between radar measurements $s_{i}$ and $s_{j}$. The pseudocovariance is then used to approximate the true covariance of $\phi_{i j}^{\text {decor }}$ and $\phi_{k l}^{\text {decor }}$ :

$$
\begin{aligned}
& \operatorname{cov}\left(\phi_{i j}^{\text {decor }}, \phi_{k l}^{\text {decor }}\right)= \\
& \frac{\sigma_{i j}}{\sqrt{1-\rho_{i j}}} \cdot \widetilde{\operatorname{cov}}\left(\phi_{i j}^{\text {decor }}, \phi_{k l}^{\text {decor }}\right) \cdot \frac{\sigma_{k l}}{\sqrt{1-\rho_{k l}}}
\end{aligned}
$$

where the scaling factor $\sigma_{i j} / \sqrt{1-\rho_{i j}}$ implies that $\operatorname{cov}\left(\phi_{i j}^{\text {decor }}, \phi_{i j}^{\text {decor }}\right)=\sigma_{i j}^{2}$. We can also derive $\gamma\left(\phi_{i j}^{\text {decor }}, \phi_{k l}^{\text {decor }}\right)$ from 6 :

$$
\gamma\left(\phi_{i j}^{\text {decor }}, \phi_{k l}^{\text {decor }}\right)=\frac{\rho_{i k}+\rho_{j l}-\rho_{i l}-\rho_{j k}}{2 \sqrt{1-\rho_{i j}} \sqrt{1-\rho_{k l}}}
$$

For ease of discussion, hereinafter we refer to the model suggested by [6] as the "Hanssen model", the model suggested by [27] as the "Agram-Simons model", and the model suggested by [28] as the "Samiei-Esfahany model". We omit discussions of models suggested in [13], [14], [26] to avoid redundancy as these models are well represented by the aforementioned three models.

\section{B. A New Model for $\gamma\left(\phi_{i j}^{\text {decor }}, \phi_{k l}^{\text {decor }}\right)$}

In the following, we present a new covariance model based on surface scattering characteristics. Consider a series of radar signals $s_{1}, s_{2}, \ldots s_{n}$ acquired at different times observing the same target. We adopt the style of analysis presented in [10]:

$$
s_{i}=\left(\sqrt{\rho_{\infty}} C+\sqrt{1-\rho_{\infty}} D_{i}\right) e^{i \psi_{i}}
$$

where $\psi_{i}$ represents propagation phases (e.g., deformation signal, atmospheric delay) at time $t_{i}, C$ represents persistent scatterers that remains coherent, and $D_{i}$ represents distributed scatterers at time $t_{i}$ that decorrelate gradually over time. For the sake of simplicity and without loss of generality, we let $C=1, E\left[D_{i}\right]=0$ and $E\left[\left|D_{i}\right|^{2}\right]=1$, so that the expected intensity of $s_{i}$ is unity. $\rho_{\infty}$ acknowledges the contribution from persistent scatterers [34]. Examining the properties of the distributed scatterers in detail,

$$
\begin{aligned}
D_{i} D_{j}{ }^{*} & =\rho_{i j}^{d}+R_{i j} \\
E\left[D_{i} D_{j}{ }^{*}\right] & =\rho_{i j}^{d}
\end{aligned}
$$

where $\rho_{i j}^{d}$ describes the correlation between distributed scatterers $D$ at times $t_{i}$ and $t_{j} . R_{i j}$ describes the remaining uncorrelated part of $D_{i} D_{j}{ }^{*}$ and has an expected value of zero. Let $z_{i j}$ represent the interferometric measurement between signals $s_{i}$ and $s_{j}$ :

$$
\begin{aligned}
z_{i j} & =s_{i} s_{j}{ }^{*} \\
& =\left(\rho_{\infty}+\left(1-\rho_{\infty}\right) D_{i} D_{j}{ }^{*}\right. \\
& \left.+\sqrt{\rho_{\infty}\left(1-\rho_{\infty}\right)}\left(D_{i}+D_{j}{ }^{*}\right)\right) e^{i\left(\psi_{i}-\psi_{j}\right)} .
\end{aligned}
$$

The correlation $\rho_{i j}$ between signals $s_{i}$ and $s_{j}$ is

$$
\begin{aligned}
\rho_{i j} & =\frac{\left|E\left[z_{i j}\right]\right|}{\sqrt{E\left[\left|s_{i}\right|^{2}\right] E\left[\left|s_{j}\right|^{2}\right]}} \\
& =\rho_{\infty}+\left(1-\rho_{\infty}\right) \rho_{i j}^{d} .
\end{aligned}
$$

Note that $\rho_{i j}^{d}$ can be of any generic form that describes temporal decorrelation of distributed scatterers. For example, with an exponential decay model $\rho_{i j}^{d}=$ $\exp \left(-\left|t_{i}-t_{j}\right| / \tau\right), \sqrt{12}$ takes the form of the generic decorrelation model presented in [34], [35].

$$
\rho(t)=\rho_{\infty}+\left(1-\rho_{\infty}\right) e^{-\frac{t}{\tau}}
$$

where $\tau$ is the characteristic correlation time of the surface. 
Substitute (9) and (12) into (11),

$$
\begin{aligned}
z_{i j} & =\left(\rho_{i j}+\left(1-\rho_{\infty}\right) R_{i j}\right. \\
& \left.+\sqrt{\rho_{\infty}\left(1-\rho_{\infty}\right)}\left(D_{i}+D_{j}{ }^{*}\right)\right) e^{i\left(\psi_{i}-\psi_{j}\right)}
\end{aligned}
$$

The first term of (14) represents coherent signals between time $t_{i}$ and $t_{j}$, which is the expected value of $z_{i j}$, the second and third terms are associated with decorrelation during this time period:

$$
\begin{aligned}
z_{i j}-E\left[z_{i j}\right] & =\left(\left(1-\rho_{\infty}\right) R_{i j}\right. \\
& \left.+\sqrt{\rho_{\infty}\left(1-\rho_{\infty}\right)}\left(D_{i}+D_{j}^{*}\right)\right) e^{i\left(\psi_{i}-\psi_{j}\right)}
\end{aligned}
$$

where $z_{i j}-E\left[z_{i j}\right]$ represents the zero-mean complex decorrelation noise component in the interferometric measurement $z_{i j}$.

The covariance of the complex decorrelation noise components in $z_{i j}$ and $z_{k l}$ is then

$$
\begin{gathered}
\operatorname{cov}\left(z_{i j}, z_{k l}\right)=E\left[\left(z_{i j}-E\left[z_{i j}\right]\right)\left(z_{k l}-E\left[z_{k l}\right]\right)^{*}\right] \\
=\left(\rho_{i k} \rho_{j l}-\rho_{\infty}{ }^{2}\right) e^{i\left(\psi_{i}-\psi_{j}-\psi_{k}+\psi_{l}\right)}
\end{gathered}
$$

Detailed derivation of $(16)$ is shown in the Appendix A. The correlation coefficient between complex interferometric measurements is therefore:

$$
\begin{aligned}
\gamma\left(z_{i j}, z_{k l}\right) & =\frac{\left|\operatorname{cov}\left(z_{i j}, z_{k l}\right)\right|}{\sqrt{E\left[\left|z_{i j}-E\left[z_{i j}\right]\right|^{2}\right] E\left[\left|z_{k l}-E\left[z_{k l}\right]\right|^{2}\right]}} \\
& =\frac{\rho_{i k} \rho_{j l}-\rho_{\infty}^{2}}{1-\rho_{\infty}^{2}}
\end{aligned}
$$

We find via numerical simulation that the relation between $\gamma\left(\phi_{i j}^{\text {decor }}, \phi_{k l}^{\text {decor }}\right)$ and $\gamma\left(z_{i j}, z_{k l}\right)$ exhibits a powerlaw behavior (See Appendix B)

$$
1-\gamma\left(\phi_{i j}^{\text {decor }}, \phi_{k l}^{\text {decor }}\right) \approx\left[1-\gamma\left(z_{i j}, z_{k l}\right)\right]^{\frac{1}{2}}
$$

Combining (17)) and (18), we have

$$
\gamma\left(\phi_{i j}^{\text {decor }}, \phi_{k l}^{\text {decor }}\right)=1-\sqrt{\frac{1-\rho_{i k} \rho_{j l}}{1-\rho_{\infty}^{2}}}
$$

Note that since we need $\rho_{i j}$ in (19), this model is only practically applicable to multi-looked interferograms in which estimates of correlation coefficients $\rho_{i j}$ can be obtained.

\section{DECORRELATION REDUCTION IN UNWRAPPED \\ INTERFEROMETRIC PHASE STACKS: A STACKING EXAMPLE}

Typical SBAS-like time-series algorithms estimate desired geophysical parameters by linearly combining a set of unwrapped interferometric phase measurements over the same resolution unit on the ground:

$$
\mathbf{P}=W \Phi
$$

where $P$ represents the estimated geophysical parameters, $W$ is the weighting or inversion matrix, and $\Phi=\left\{\phi_{i j}\right\}$ is the set of unwrapped interferometric phases involved in the estimation.

Each $\phi_{i j}$ can be represented as the sum of deformation, atmospheric noise and decorrelation noise [4], [18], [19]

$$
\phi_{i j}=\phi_{i j}^{d e f}+\phi_{i j}^{a t m}+\phi_{i j}^{d e c o r}
$$

Note that we have not included phase noise terms such as digital elevation model (DEM) error or thermal noise because they are either deterministic terms that can be reasonably well modeled and removed, or typically negligible uncorrelated noise terms. We have also omitted an integer ambiguity term that accounts for phase unwrapping errors. We assume that phase unwrapping is performed consistently and accurately in space and time. Modeling of phase unwrapping errors requires detailed mapping of terrain-dependent back-scatterers and is beyond the scope of this manuscript. Deformation is a deterministic process. Atmospheric noise and decorrelation noise, on the other hand, are stochastic variables with assumed zero means and are independent of each other because they represent unrelated physical processes. Therefore, the covariance matrix for a set of interferometric measurements $\Phi=\left\{\phi_{i j}\right\}$ can be expressed as:

$$
\operatorname{Cov}(\Phi)=\operatorname{Cov}\left(\Phi^{a t m}\right)+\operatorname{Cov}\left(\Phi^{\text {decor }}\right)
$$

The covariance matrix for atmospheric noise is well researched [7], [9], [36]. In this paper, we focus on the covariance matrix for decorrelation phase $\operatorname{Cov}\left(\Phi^{\text {decor }}\right)$. Assuming no atmospheric noise is present, we can propagate measurement uncertainties to uncertainties in the estimated parameters:

$$
\operatorname{Cov}(P)=W \operatorname{Cov}\left(\Phi^{\text {decor }}\right) W^{T}
$$

So far we have described four models for $\operatorname{Cov}\left(\Phi^{\text {decor }}\right)$ : the Hanssen model, the Agram-Simons model, the Samiei-Esfahany model and the proposed model. In this section, we illustrate their differences by feeding each model into (23) and then comparing 
their respective predictions of $\operatorname{Cov}(P)$. To achieve that, we need to first specify $P$ and $W$. While there are many different deformation scenarios and time-series processing schemes in real applications, we find that a simple stacking exercise over a transient event is sufficient for our purposes.

\section{A. Simulation Setting: stacking over a transient event}

Consider a radar target that decorrelates over time. Assume that a transient deformation event took place and we have $M$ consecutive radar measurements acquired over the surface before the event $s_{1}, \ldots, s_{M}$ and $M$ measurements acquired after the event $s_{M+1}, \ldots, s_{2 M}$. Therefore, we have a total of $M^{2}$ interferograms that span the transient event:

$$
\left\{\phi_{1, M+1}, \ldots, \phi_{1,2 M}, \ldots \phi_{M, M+1}, \ldots \phi_{M, 2 M}\right\}
$$

Out of these $M^{2}$ interferograms, there is a maximum of $M$ interferograms formed using unique pairs of radar measurements. One such combination is

$$
\left\{\phi_{1, M+1}, \phi_{2, M+2}, \ldots, \phi_{M, 2 M}\right\} \text {. }
$$

The $M$ interferograms with unique pairs of radar measurements are often referred to as "independent interferograms" in literature in the context of atmospheric noise mitigation [7], [36], [37]. Since these interferograms are not necessarily truly independent, we adopt the term "non-repeating stack" instead. In contrast, we refer to the previous group of measurements with all possible measurements as a "repeating stack". Note that in practice, a repeating stack does not have to include all available interferograms. Assuming no atmospheric noise is present,

$$
\phi_{i j}=\phi^{d e f}+\phi_{i j}^{d e c o r}
$$

where $\phi^{\text {def }}$ corresponds to phase change caused by the transient event and is present in every interferogram that span the event.

To retrieve signals associated with the transient deformation event $\phi^{d e f}$, we can average either stack. Both stacking strategies are widely adopted in literature [37][41]. By stacking a non-repeating stack, we have

$$
\begin{aligned}
\Phi_{n r p} & =\left[\phi_{1, M+1}, \phi_{2, M+2}, \ldots, \phi_{M, 2 M}\right]^{\prime} \\
W & =\left[\frac{1}{M} \frac{1}{M} \ldots \frac{1}{M}\right] \\
P & =W \Phi_{n r p}
\end{aligned}
$$

Similarly, by stacking a repeating stack, we have

$$
\begin{aligned}
\Phi_{r p} & =\left[\phi_{1, M+1}, \ldots, \phi_{1,2 M}, \ldots \phi_{M, M+1}, \ldots \phi_{M, 2 M}\right]^{\prime} \\
W & =\left[\frac{1}{M^{2}} \frac{1}{M^{2}} \ldots \frac{1}{M^{2}}\right] \\
P & =W \Phi_{r p}
\end{aligned}
$$

\section{B. Construction of $\operatorname{Cov}\left(\Phi^{\text {decor }}\right)$}

We are interested in determining $\sigma^{2}(P)$ for both stacks. Adopting the Cramer-Rao bound [33]

$$
\sigma^{2}\left(\phi_{i j}\right)=\frac{1-\rho_{i j}^{2}}{2 \rho_{i j}^{2}}
$$

as phase variances, we construct $\operatorname{Cov}\left(\Phi^{\text {decor }}\right)$ using (1) with $\gamma\left(\phi_{i j}^{\text {decor }}, \phi_{k l}^{\text {decor }}\right)$ given by each of the four models.

We also need to specify a temporal decorrelation model $\rho(t)$. Here we choose the generic decorrelation model (13). The parameters in (13) are highly dependent on land covers, wavelength and climate [34], [35]. In this simulation, we examine the impacts of correlation time constant $\tau$ and persistent correlation $\rho_{\infty}$ on $\operatorname{Cov}\left(\Phi^{\text {decor }}\right)$. We also examine the impacts of different numbers of radar measurements $(2 M)$, and acquisition intervals on the performance of decorrelation noise reduction.

\section{Comparison Between Existing And The Proposed Decorrelation Covariance Models}

Fig. 1 depicts the predictions of $\sigma^{2}(P)$ from both the existing and the proposed models for surfaces with varying decorrelation rates and fixed persistent coherence $\left(\rho_{\infty}=0.1\right)$. We use the ratio between characteristic correlation time $\tau$ and SAR acquisition interval $\Delta t$ to represent apparent surface correlation time - higher ratio means slower apparent decorrelation. We set $M=25$. The Hanssen model (green lines) predicts larger phase variance with non-repeating stacking regardless of surface decorrelation rates; the Agram-Simons model (blue lines) predicts zero or small differences between two stacking strategies with either rapidly or very slowly decorrelating surfaces; and both the Samiei-Esfahany model (magenta lines) and the proposed model (red lines) predict significantly higher noise reduction from repeating stacking when $\tau / \Delta t<15$ and similar noise reduction performances from either stacking strategy when $\tau / \Delta t>15$. Additionally, we observe that: 


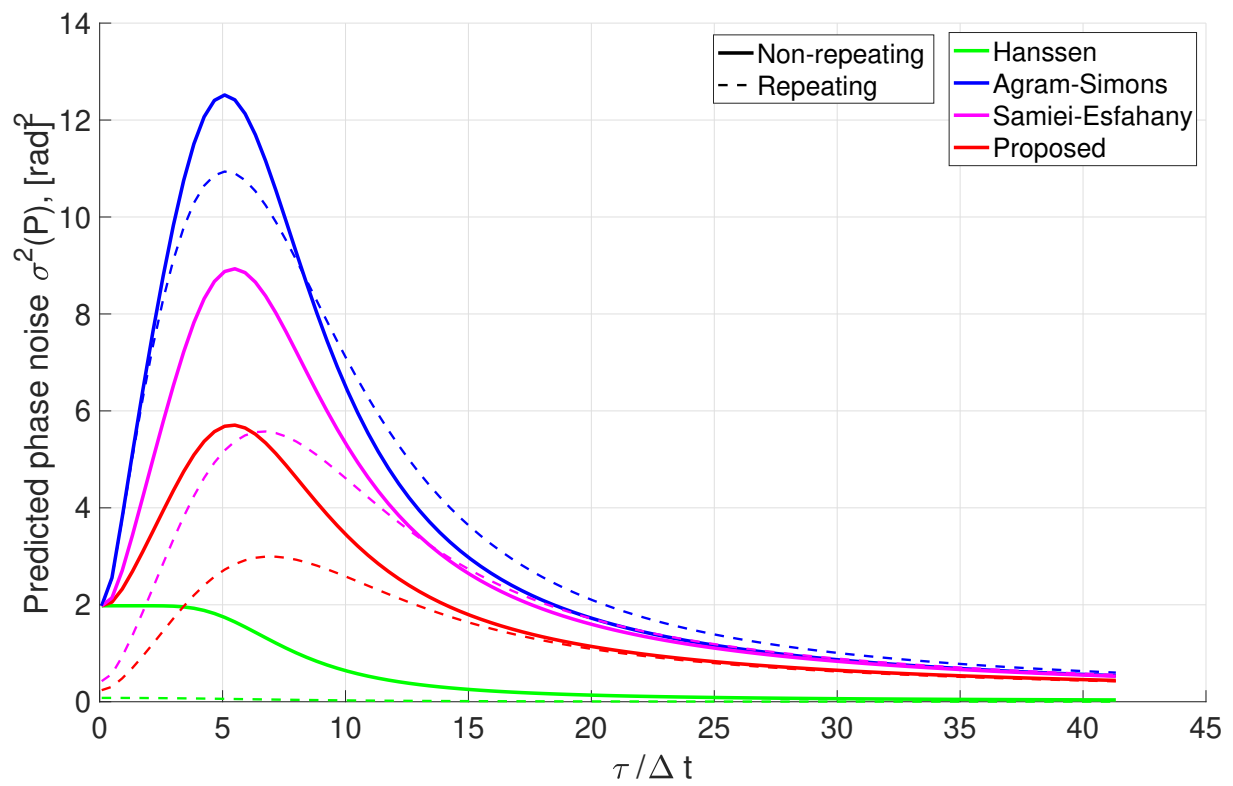

Fig. 1. Predicted uncertainty associated with decorrelation noise after non-repeating stacking and repeating stacking using the Hanssen model, the Agram-Simons model, the Samiei-Esfahany model and the proposed model.

1. The Agram-Simons model predicts the highest phase noise (i.e., the least reduction in decorrelation noise) for both non-repeating and repeating stacking. This means that the Agram-Simons model suggests high correlation between decorrelation noise components. In contrast, the Hanssen model, which assumes independence between decorrelation noise components, predicts the lowest uncertainty. The Samiei-Esfahany model suggests higher correlation than that suggested by the proposed model, but lower than that suggested by the Agram-Simons model (within the range of $\tau / \Delta t$ plotted in Fig 1 .

2. Predictions from the Samiei-Esfahany, the AgramSimons model and the proposed model model all show a peak around $\tau / \Delta t \approx 6$ while predictions from the Hanssen model shows a steady decrease with increasing apparent correlation time $\tau / \Delta t$. Two factors influence the predicted phase variance: a) the average phase noise level in interferograms and b) the degree of correlation between decorrelation noise components. The higher the average phase noise, the higher the predicted phase variance; the higher the degree of correlation between decorrelation noise terms, the higher the predicted phase variance. When $\tau / \Delta t$ is small, correlation between decorrelation noise terms are negligible in all three models, and when $\tau / \Delta t$ is large, the average phase noise level is low. Therefore the predicted phase variances in all three models are low at both ends of $\tau / \Delta t$ but high in the middle. Since there is no correlation in the Hanssen model, the predicted phase variance decreases monotonically with $\tau / \Delta t$.

Fig. 2 depicts how varying $\rho_{\infty}$ or the number of SAR measurements $(2 M)$ impacts predicted phase noises with respect to different apparent decorrelation rates $\tau / \Delta t$. Fig. 2 (a) shows that increasing $\rho_{\infty}$ decreases the dependency of predicted uncertainty on surface decorrelation rate as contributions from persistent scatterer increases. Fig. 2 (b) illustrates that for a slowly decorrelating surface $(\tau / \Delta t=10)$, increasing $M$ increases predicted uncertainties while for a rapidly decorrelating surface $(\tau / \Delta t=0.1)$, increasing $M$ reduces predicted uncertainty. Increasing $M$ is equivalent to increasing the observation window length. For rapidly decorrelating surfaces, longer observational window provides additional measurements that are at a comparable noise level (determined by $\rho_{\infty}$ ) with the original stack. Since these measurements are almost independent with the original stack, increasing $M$ reduces noise. In contrast, for slowly decorrelating surfaces, long temporal span interferograms provide highly correlated measurements at a higher noise level. Therefore increasing $M$ increases noise. The contrasting impacts of $M$ with respect to surface decorrelation rates again reflect the two factors that influence the predicted phase variance: 1) the average phase noise level in interferograms and 2) the degree of correlation between 


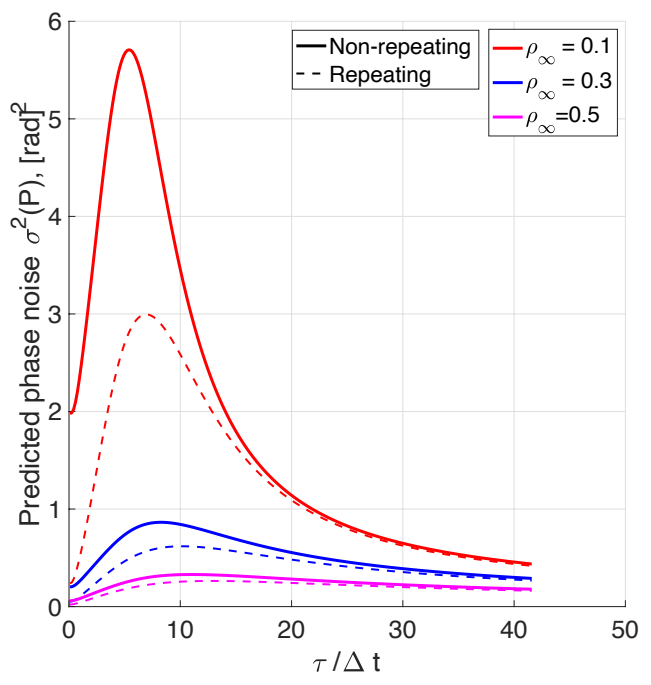

(a)

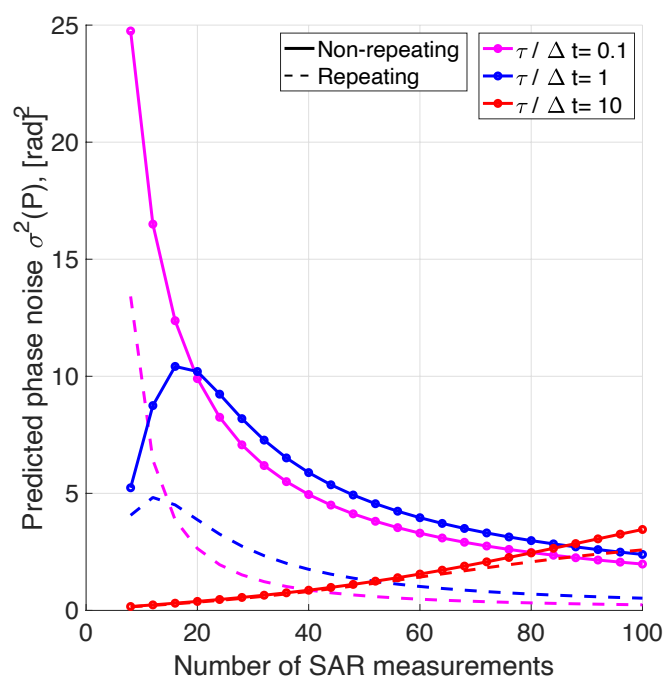

(b)

Fig. 2. The influence of (a) $\rho_{\infty}$ and (b) the number of SAR measurements with respect to different apparent decorrelation rates on predicted uncertainty using the proposed model.

decorrelation noise terms. Increasing $M$ raises average noise level in interferograms but reduces the degree of correlation between decorrelation noise components. Therefore, when SAR measurements are sampled at a comparable interval with the surface correlation time $(\tau / \Delta t=1)$, the predicted uncertainty first increases then decreases when increasing $M$.

\section{Implication for stacking strategies}

Ultimately our goal is to effectively reduce decorrelation noise. Knowledge of $\operatorname{Cov}\left(\Phi^{\text {decor }}\right)$ can help us determine optimal processing strategies. In our simple example of stacking over a transient event, we compared the performances of two common stacking practices: non-repeating stacking and repeating stacking. If the target decorrelates exponentially over time, Fig 1 provides predictions of performance of either stack on decorrelation reduction with the given configuration of $M=25$ and $\rho_{\infty}=0.1$. For example, if radar measurements are not sampled 10 times the rate of surface decorrelation $(\tau / \Delta t<10)$, the proposed model suggests use of repeating stack because the performance differences between the two stacks are significant. On the other hand, if $\tau / \Delta t>10$, the performance differences between the two stacks is insignificant, non-repeating stack becomes a better choice because it involves computation of fewer interferograms and hence is more efficient. Fig. 2 (b) demonstrates that for rapidly decorrelating surfaces, it is preferable to have long observation windows, though the rate of noise reduction declines with increasing $M$. In comparison, for surfaces that exhibit slow decorrelation rates, a small subset of interferometric pairs is sufficient to achieve satisfactory results.

In real applications, the weighting matrix $W$ can take on more complicated forms than the ones shown in 25) and (26). Moreover, there are often constraints on $M$. Nevertheless the same procedure as described above can be followed to guide the choice of $W$ and the set of interferometric pairs to be used.

\section{VALIDATION With REAL DATA}

In this section, we compare and assess all four decorrelation phase covariance models - the Hanssen model, the Samiei-Esfahany model, the Agram-Simons model, and the proposed model - with C-band Sentinel1 data collected in both Cascadia and Death Valley. Similar to Section III, we construct $\operatorname{Cov}\left(\phi_{i j}^{\text {decor }}, \phi_{k l}^{\text {decor }}\right)$ using 11 with $\gamma\left(\phi_{i j}^{\text {decor }}, \phi_{k l}^{\text {decor }}\right)$ given by each of the four models. Note that we adopt the correlation forms of the models to avoid estimation bias caused by phase variance estimation - the Samiei-Esfahany model uses the Cramer-Rao bound to estimate phase variances while all three other models use observed phase variances. Since the Cramer-Rao bound only holds for high coherence targets and hence underestimate 
(a)

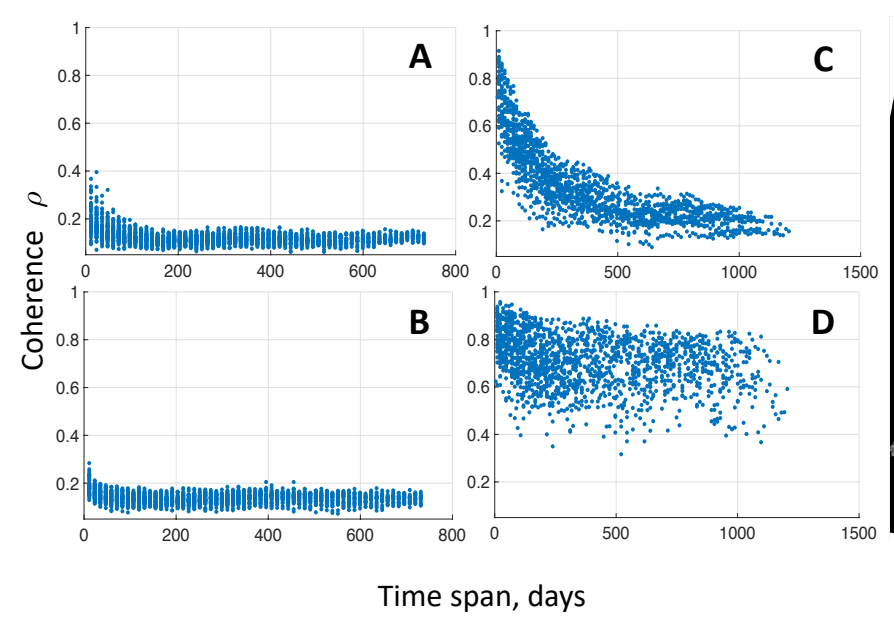

(b)

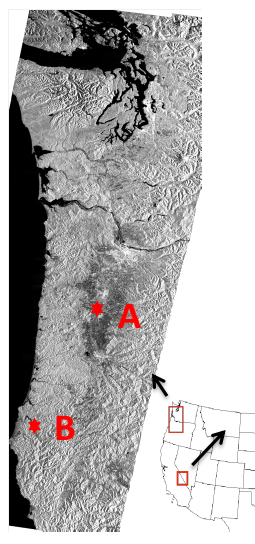

(c)

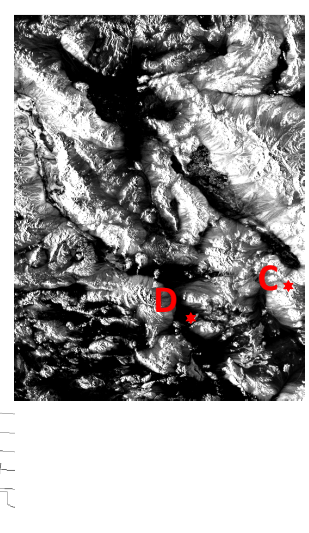

Fig. 3. Examples of observed temporal variations of correlation in the Cascadia and the Death Valley regions. (a) Scatter plots of correlation vs time in locations A, B, C and D. (b) A and B are located in the heavily vegetated Cascadia region, (c) C and D are located in the desert of Death Valley. A and B both exhibit a characteristic decorrelation time of roughly 30 days and an asymptotic coherence $\rho_{\infty}$ of 0.1 . C and $\mathrm{D}$, on the other hand, show much longer decorrelation time of approximately 400 days and 200 days and higher asymptotic coherence $\rho_{\infty}$ of 0.2 and 0.6 , respectively.

phase variances of the stacks, we modify the original Samiei-Esfahany model (3) by adopting observed phase variances instead. We estimate observed phase variances of individual interferograms $\sigma^{2}\left(\phi_{i j}\right)$, and of averaged stacks $\sigma^{2}\left(\phi_{n r p}^{\text {decor }}\right), \sigma^{2}\left(\phi_{r p}^{\text {decor }}\right)$ by calculating phase variances inside 50 pixel by 50 pixel boxes (15 by $15 \mathrm{~km}^{2}$ in area), assuming the spatial variances of atmospheric noise and phase unwrapping errors are negligible in the box. It is reasonable to assume that areas with moderate to high correlations are reliably unwrapped. Low correlation areas, on the other hand, are prone to phase unwrapping errors. We assume that phase unwrapping errors are either uniform or sparsely distributed inside a small estimation window and hence contribute little to the overall phase variance.

Similar to section III, we form both non-repeating and repeating stacks that span over a given time interval in both regions, and predict residual decorrelation noise after averaging each stack using both the proposed and existing decorrelation phase covariance models. We estimate $\rho_{i j}$ using sampled local averages over 300 meter by 300 meter windows. We then compare predicted residual decorrelation noise with observed residual decorrelation noise.

Both Cascadia and Death Valley exhibit exponential decay in correlation with time, but with contrasting decorrelation rates (Fig. 3). For the Cascadia region, we form a non-repeating stack consisting of 40 interferograms and a repeating stack consisting of 1600 interferograms. For the Death Valley region, we form a non-repeating stack consisting of 28 interferograms and a repeating stack consisting of 784 interferograms. By averaging the respective stacks, we obtained $\phi_{n r p}$ and $\phi_{r p}$ for both regions.

TABLE I

AVERAGE PREDICTION ERROR FOR EACH MODEL OVER TWO SURFACES. WE DEFINE PREDICTION ERRORS AS THE DISTANCES (UNIT: $[\mathrm{rad}]^{2}$ ) BETWEEN POSITIONS OF DATA POINTS (BEIGE CIRCLES) AND POSITIONS OF THEIR CORRESPONDING PREDICTION POINTS (PURPLE STARS, ORANGE SQUARES, CYAN DIAMONDS OR MAGENTA INVERTED TRIANGLES) IN FIG. 4.

\begin{tabular}{l|cc}
\hline Models & Cascadia & Death Valley \\
\hline Agram-Simons & 0.300 & 0.368 \\
\hline Hanssen & 0.097 & 0.114 \\
\hline Samiei-Esfahany (Modified) & 0.096 & 0.231 \\
\hline Proposed & 0.092 & 0.105 \\
\hline
\end{tabular}

Comparisons between phase variances associated with $\phi_{n r p}$ and $\phi_{r p}$ are shown in Fig. 4 (a) and (b). We obtained $\sigma^{2}\left(\phi_{n r p}^{\text {decor }}\right)$ and $\sigma^{2}\left(\phi_{r p}^{\text {decor }}\right)$ at evenly distributed image grid points. For the Cascadia region ( $\tau / \Delta t \approx 1$ or 2 ), the repeating stack yields smaller uncertainties - on average $0.037 \mathrm{rad}^{2}$ ) than the non-repeating stack - on average $0.284 \mathrm{rad}^{2}$ (Fig. 4 (a), beige circles), confirming predictions from the proposed model. For the Death Valley region where the apparent correlation time $\tau / \Delta t>20$, non-repeating 

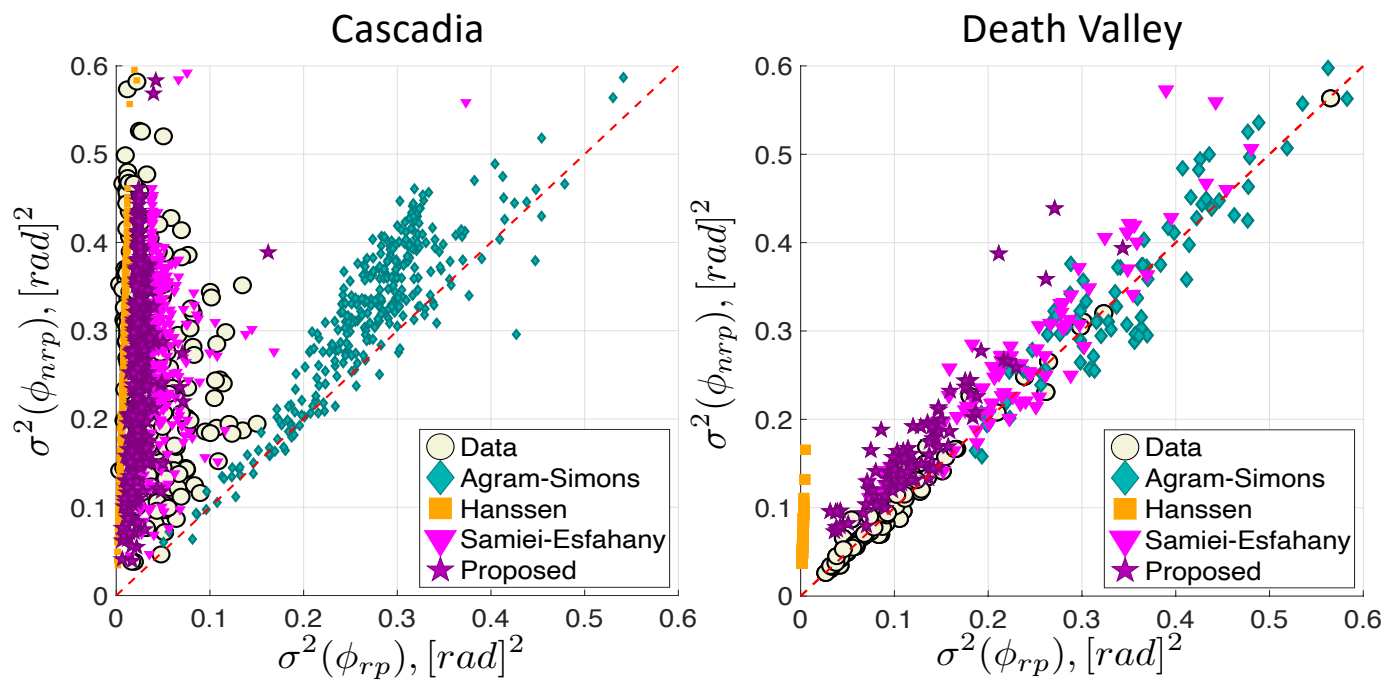

(a)

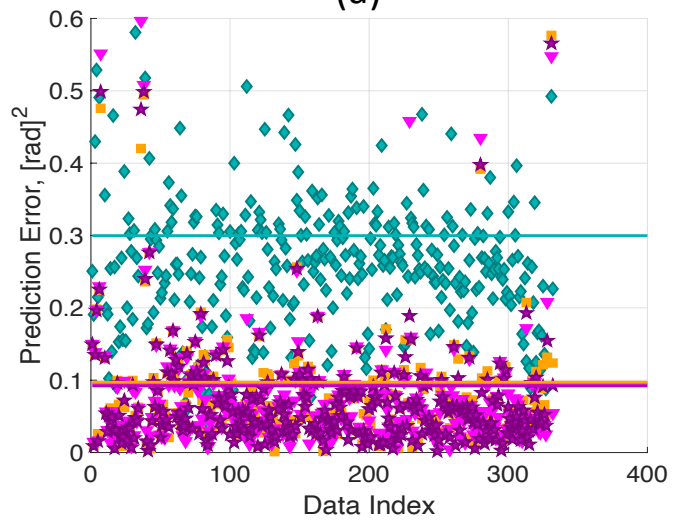

(c)

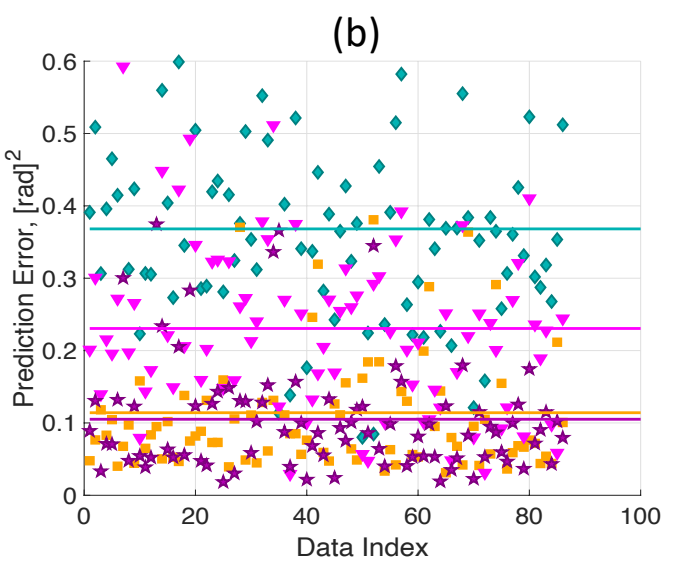

(d)

Fig. 4. Predicted phase variance and prediction errors after non-repeating stacking vs repeating stacking in (a) (c) the Cascadia region and (b) (d) the Death Valley region. Data (circles) show that for the Cascadia region, repeating stacking produces smaller phase variances and for the Death Valley region, similar phase variances, as compared to non-repeating stacking. Model predictions are shown in (a) and (b) while their respective errors are shown in (c) and (d) with the average prediction errors marked by solid lines. Predictions from the proposed model (stars) match the data best in both cases. The accuracy of each model prediction is summarized in Table [

and repeating stacking produce comparable results - on average $0.105 \mathrm{rad}^{2}$ and $0.102 \mathrm{rad}^{2}$, respectively (Fig. 4 (b), beige circles), which are also consistent with predictions from the proposed model.

Predictions from both existing and the proposed models are shown in Fig. 4 and Table. I] Predictions from the proposed model (purple stars) match best with actual observations (beige circles). Predictions from existing models are consistent with the simulation results in Section III. For example, in Cascadia where the decorrelation rate is rapid, all models give comparable estimates for the non-repeating stack, while the Agram-Simons model (cyan diamonds) and the modified Samiei-Esfahany model (magenta triangles) give higher, the Hanssen model (orange squares) gives lower estimates of phase variances for the repeating stack than the proposed model. In the Death Valley region where the decorrelation rate is on the order of years, all models except for the Hanssen model predict comparable phase variances between the non-repeating and the repeating stack.

Finally, we use an example to illustrate the significant reduction in decorrelation noise offered by including repeating interferograms in the stack over rapidly decorrelating areas. Fig. 5(a) and (b) depicts stacks over a slow slip deformation event [42], [43] that occurred in February 2016 in the Cascadia region. As a comparison, non-repeating and repeating stacking results in Death 
Valley (Fig[5 (c) and (d)) show minimal differences. Slow slip events are usually hard to capture in the Cascadia region with InSAR due to extremely low signal to noise ratios. Atmospheric noise and decorrelation are the two main limitation factors. With 10 Sentinel-1 SAR acquisitions before the slow slip event and 10 Sentinel1 SAR acquisitions after, we formed a non-repeating interferogram stack consisting of 10 interferograms and a repeating stack consisting of 100 interferograms. It is apparent that the result from using repeating stacking yields a much cleaner signal pattern. Fig. 6 compares phase measurements along four profile lines between non-repeating and repeating stacking. Again, it is clear that while both stacking strategies produce measurements of the same expected signal, repeating stacking produces measurements with $80 \%$ less phase variance - the uncertainty associated with decorrelation reduces from $0.33 \mathrm{~cm}$ to $0.13 \mathrm{~cm}$ in the line-of-sight direction. With the same number of independent acquisitions, atmospheric noise is reduced to the same extent after either non-repeating stacking or repeating stacking. Therefore, the significant reduction in phase noise reflects reduced decorrelation noise in the repeating stack. It is worth noting that repeating stacking may also contribute to reduction of phase unwrapping errors. Fig. 8 (Appendix C) shows that phase unwrapping errors are more prevalent in longer temporal baseline interferograms. The problem of phase unwrapping error reduction is analogous to that of decorrelation noise reduction. On one hand, a repeating stack may reduce phase unwrapping errors more because of its superior number of measurements. On the other hand, a repeating stack includes more long temporal baseline interferograms that are susceptible to phase unwrapping errors. While detailed statistical models for phase unwrapping errors are beyond the scope of this work, a reasonable assumption for phase unwrapping errors in rapidly decorrelating areas are to treat them as random variables, i.e., the amount of phase unwrapping errors in a particular pixel vary independently in different interferograms. Since decorrelation noise can be treated as an independent noise term for rapidly decorrelating surfaces, the addition of another random noise term does not alter the statistical description for overall phase noise. Therefore, in the Cascadia case, repeating stacking performs better in reducing overall phase noise than nonrepeating stacking, as expected from the proposed model.

\section{DISCUSSIONS AND CONCLUSIONS}

We have described a new decorrelation phase covariance model for unwrapped interferogram phase stacks and compared the proposed model with three existing models - the Hanssen model, the SamieiEsfahany model and the Agram-Simons model. Validations with Sentinel-1 data collected in both the Cascadia region and Death Valley region show that our model best captures decorrelation noise propagation in interferogram stacks. We demonstrated with a simple stacking exercise that the proposed decorrelation phase covariance model can facilitate choice of SBAS-like time-series processing strategies. The workflow applied to the stacking example can be easily adapted to general SBAS-like time-series algorithms in three steps:

1 Obtain the temporal decorrelation characteristics $\rho(t)$ in the area of interest. In some cases we may have a prior knowledge or a good estimation of $\rho(t)$. Otherwise, estimate $\rho(t)$ from the processed stack.

2 Construct the decorrelation phase covariance matrix with (1) and (19) for the entire interferometric phase stack $\Phi$. Phase variances can be estimated using the PDF of interferometric phases [2], [29]-[32].

3 Estimate residual decorrelation noise $C O V(P)$ using (23) with different weighting matrices $W$. Determine the optimal $W$ and subset of interferometric pairs $\Phi$ in terms of effective noise reduction and efficiency.

As we head into an era with an ever-growing SAR archive, understanding and quantifying uncertainties associated with decorrelation noise is of critical importance. A rigorous, comprehensive noise covariance model allows the InSAR community to better assess uncertainties with InSAR measurements, and to extend InSAR applications from mid-to-high correlation areas to low-correlation areas.

\section{APPENDIX A \\ DERIVATION OF (16)}

We model the distributed scatterers $D_{i}$ as complex Gaussian random variables. $D_{i}$ 's can be represented as

$$
D_{i}=\rho_{i-1, i}^{d} D_{i-1}+\sqrt{1-\rho_{i-1, i}^{d}} n_{i}
$$

where $n_{i}$ is a complex Gaussian random variables with an expected intensity of unity, and that is uncorrelated with $D$ 's and $n$ 's other than itself. With (27), we can derive that

$$
\begin{aligned}
\rho_{i j}^{d} \rho_{j k}^{d} & =\rho_{i k}^{d} \\
E\left(D_{i} D_{j}^{*}\right) & =\rho_{i j}^{d} \\
E\left(\left|D_{i}\right|^{2}\left|D_{j}\right|^{2}\right) & =1+\rho_{i j}^{d}{ }^{2}
\end{aligned}
$$


(a)

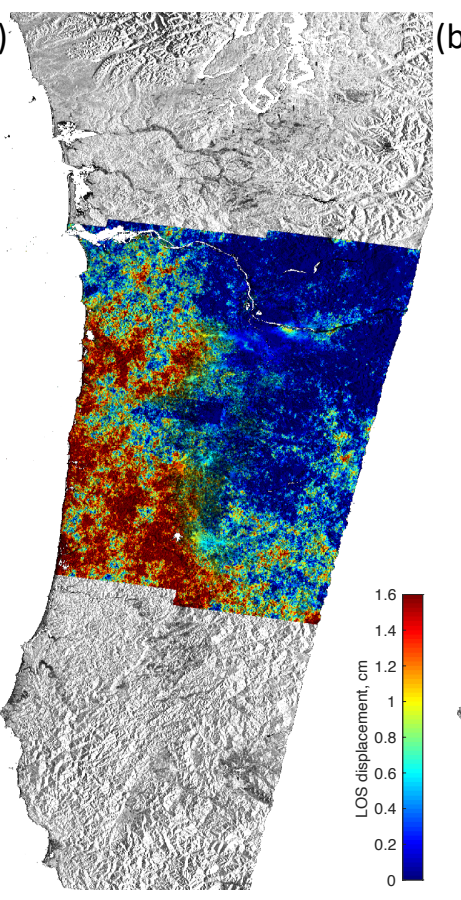

(b)

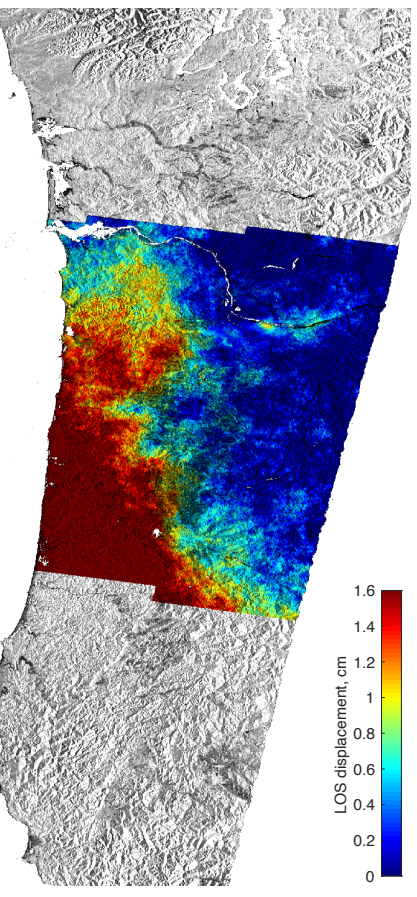

(c)

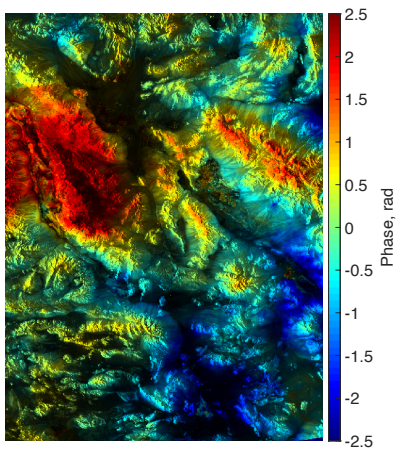

(d)

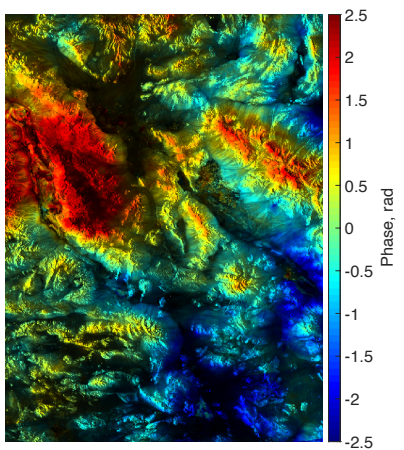

Fig. 5. Results from (a) non-repeating and (b) repeating stacking over the February 2016 slow slip event in the Cascadia region. The result from repeating stacking suffers from less decorrelation noise than the result from non-repeating stacking. Areas with less coverage than 20 acquisitions are masked. Phase measurements are converted to radar line-of-sight (LOS) measurements. Results from (c) non-repeating and (d) repeating stacking in the Death Valley region. The differences between the two stacking results are minimal.

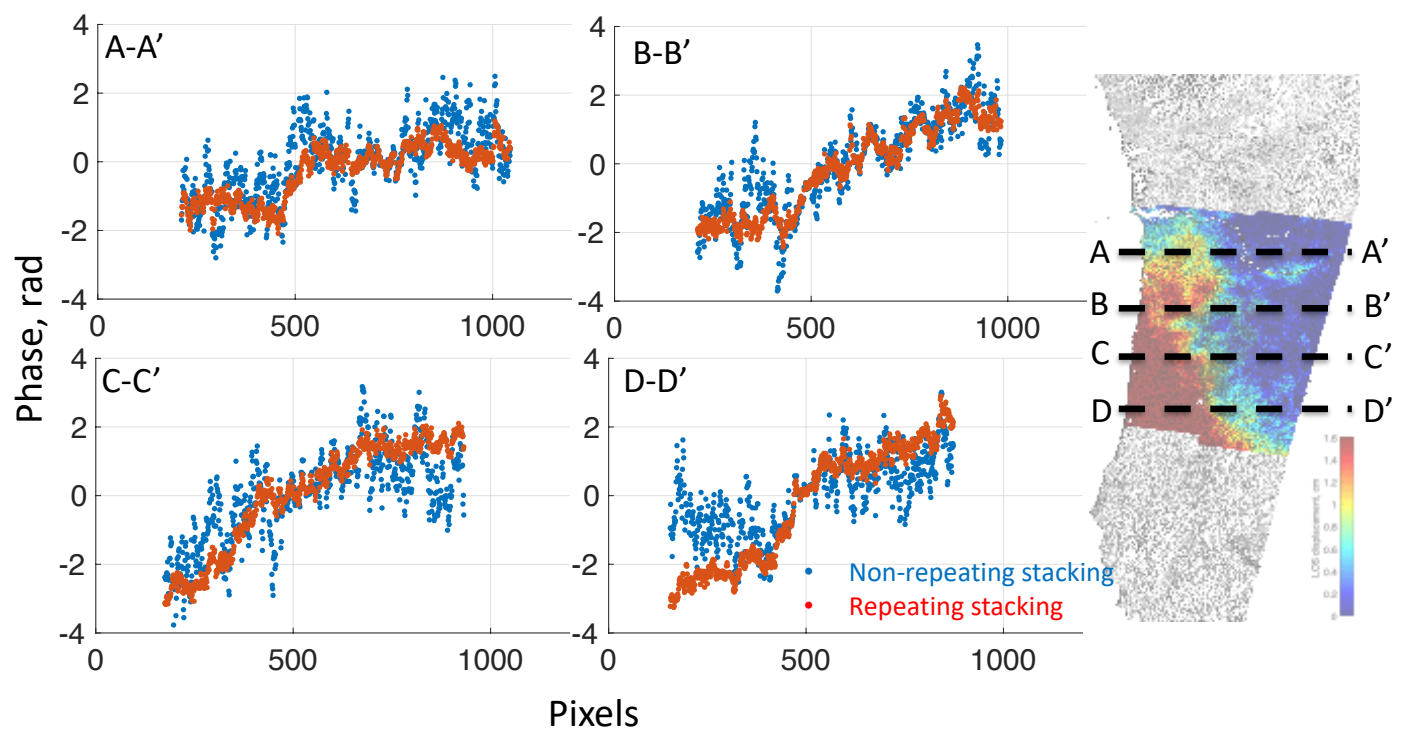

Fig. 6. Comparisons between non-repeating and repeating stacking results over profile lines A-A', B-B', C-C' and D-D'. Repeating stacking (red dots) produces similar mean measurements as with non-repeating stacking (blue dots), but with much 83\% less phase variances. Equivalently, repeating stacking produces measurements with $0.33 \mathrm{~cm}$ line-of-sight uncertainties compared to $0.13 \mathrm{~cm}$ after non-repeating stacking. 


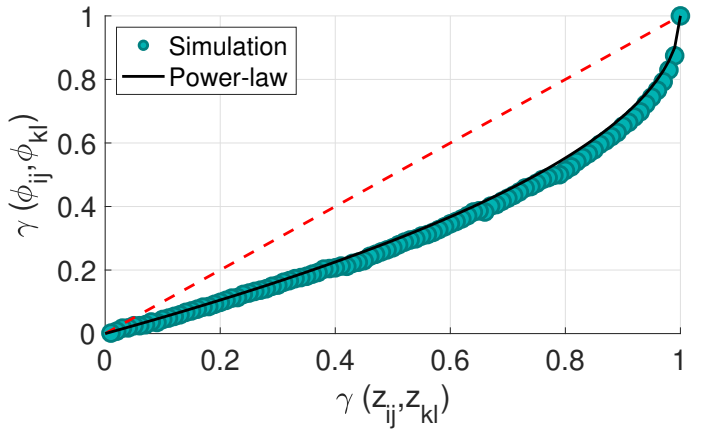

Fig. 7. The relation between $\gamma\left(z_{i j}, z_{k l}\right)$ and $\gamma\left(\phi_{i j}, \phi_{k l}\right)$ exhibits a power-law behavior $1-\gamma\left(\phi_{i j}^{\text {decor }}, \phi_{k l}^{\text {decor }}\right) \approx\left[1-\gamma\left(z_{i j}, z_{k l}\right)\right]^{\frac{1}{2}}$.

Now we can derive (16):

$$
\begin{aligned}
\operatorname{cov} & \left(z_{i j}-E\left[z_{i j}\right], z_{k l}-E\left[z_{k l}\right]\right) \\
& =E\left[\left(z_{i j}-E\left[z_{i j}\right]\right)\left(z_{k l}-E\left[z_{k l}\right]\right)^{*}\right] \\
& =E\left\{\left(1-\rho_{\infty}\right)^{2} R_{i j} R_{k l}^{*}\right. \\
& +\rho_{\infty}\left(1-\rho_{\infty}\right)\left(D_{i} D_{k}^{*}+D_{i} D_{l}+D_{j}^{*} D_{k}^{*}+D_{j}^{*} D_{l}\right) \\
& +\left(1-\rho_{\infty}\right) \sqrt{\rho_{\infty}\left(1-\rho_{\infty}\right)}\left(R_{i j}\left(D_{k}^{*}+D_{l}\right)\right. \\
& \left.\left.+R_{k l}^{*}\left(D_{i}+D_{j}^{*}\right)\right)\right\} e^{i\left(\psi_{i}-\psi_{j}-\psi_{k}+\psi_{l}\right)} \\
& =\left(\left(1-\rho_{\infty}\right)^{2} E\left(R_{i j} R_{k l}^{*}\right)\right. \\
& \left.+\rho_{\infty}\left(1-\rho_{\infty}\right)\left(\rho_{i k}^{d}+\rho_{j l}^{d}\right)\right) e^{i\left(\psi_{i}-\psi_{j}-\psi_{k}+\psi_{l}\right)} \\
& =\left(\left(1-\rho_{\infty}\right)^{2} \rho_{i k}^{d} \rho_{j l}^{d}\right. \\
& \left.+\rho_{\infty}\left(1-\rho_{\infty}\right)\left(\rho_{i k}^{d}+\rho_{j l}^{d}\right)\right) e^{i\left(\psi_{i}-\psi_{j}-\psi_{k}+\psi_{l}\right)} \\
& =\left(\rho_{i k} \rho_{j l}-\rho_{\infty}^{2}\right) e^{i\left(\psi_{i}-\psi_{j}-\psi_{k}+\psi_{l}\right)}
\end{aligned}
$$

\section{APPENDIX B}

Numerical Simulation of (18)

To find the relation between $\gamma\left(z_{i j}, z_{k l}\right)$ and $\phi_{i j}, \phi_{k l}$, we simulate a series of complex Gaussian random variables with decreasing correlation from 1 to 0 . We then compute the corresponding correlations between phases of these complex Gaussian random variables (Fig. 7).

\section{APPENDIX C}

\section{SAMPLE INTERFEROGRAMS OF THE CASCADIA STACK}

The non-repeating stack of Cascadia consists of 10 interferograms that have similar temporal baselines (on average 272 days). Fig. 8 (b) and (e) show wrapped and unwrapped phase from a non-repeating stack interferogram with a temporal baseline of 264 days. The repeating stack of Cascadia consists of 100 interferograms, which includes interferograms with temporal baseline ranging from 48 days to 480 days. Fig. 8 (a) and (d) show wrapped and unwrapped phases from an interferogram with a temporal baseline of 48 days. Fig. 8 (c) and (f) show wrapped and unwrapped phases from an interferogram with a temporal baseline of 480 days. Phase unwrapping errors manifest as jumps of $2 \pi$ radians. Fig. 8 shows that phase unwrapping errors are more prevalent in longer temporal baseline interferograms.

\section{ACKNOWLEDGMENT}

We would like to thank the anonymous reviewers for their constructive criticisms that greatly improved the quality of this manuscript. This work was supported by NASA Earth Science and Interior Grant NNX 17AE036. Copernicus Sentinel-1 data 2015-2018 was retrieved from ASF DAAC 7 May 2018, processed by ESA, https://www.asf.alaska.edu. 

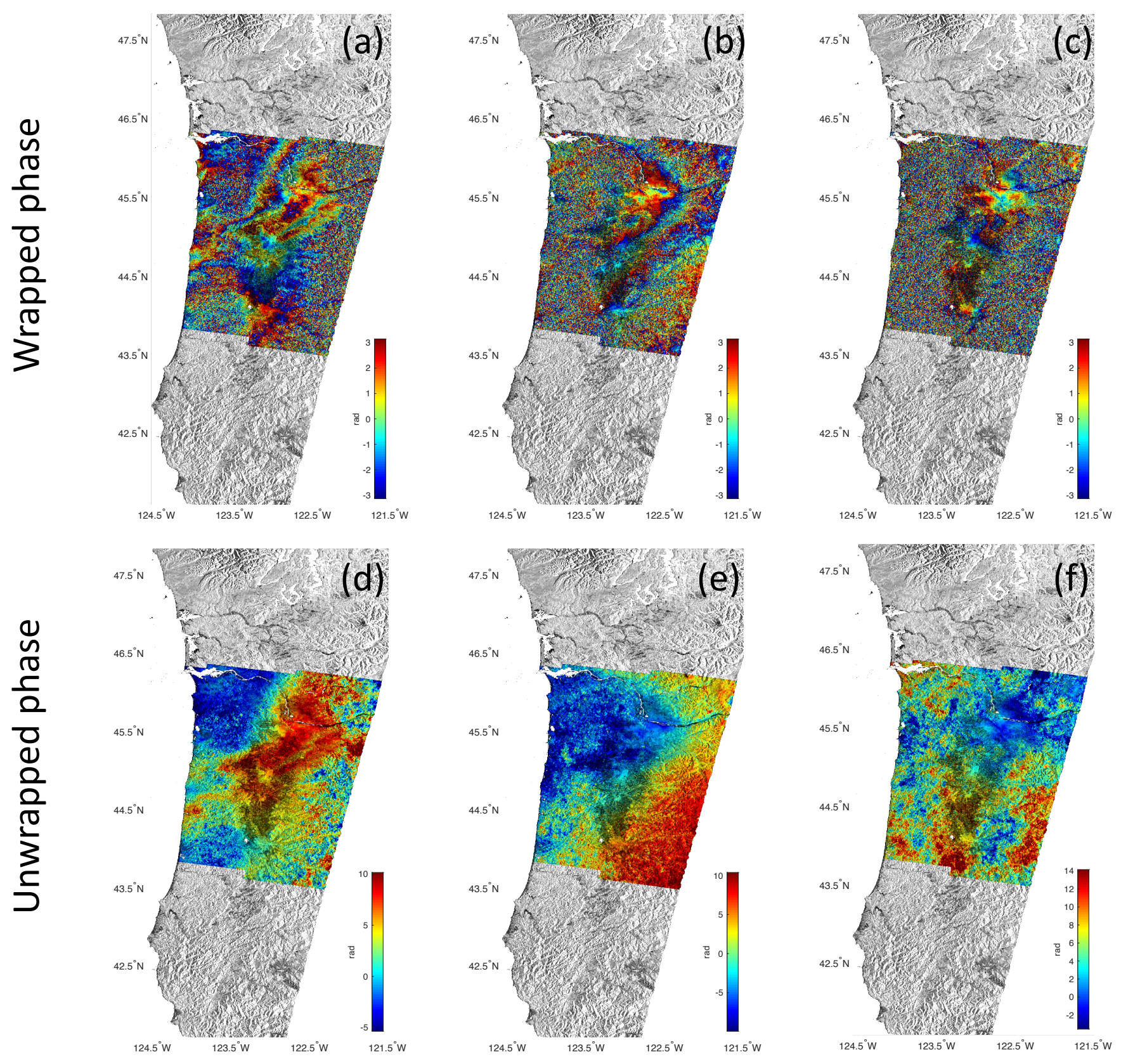

Fig. 8. Three sample interferograms before and after unwrapping. (a)(d) A sample interferogram with 48 days temporal baseline (b)(e) A sample interferogram with 264 days temporal baseline (c)(f) A sample interferogram with 480 days temporal baseline. Phase unwrapping errors manifest as jumps/spikes of $2 \pi$ rad. Phase unwrapping errors are more prevalent in longer temporal baseline interferograms.

\section{REFERENCES}

[1] H. A. Zebker, P. A. Rosen, R. M. Goldstein, A. Gabriel, and C. L. Werner, "On the derivation of coseismic displacement fields using differential radar interferometry: The landers earthquake," Journal of Geophysical Research: Solid Earth, vol. 99, no. B10, pp. 19617-19634, 1994.

[2] R. Bamler and P. Hartl, "Synthetic aperture radar interferometry," Inverse problems, vol. 14, no. 4, p. R1, 1998.

[3] P. A. Rosen, S. Hensley, I. R. Joughin, F. Li, S. N. Madsen, E. Rodriguez, and R. M. Goldstein, "Synthetic aperture radar interferometry," 1998.

[4] R. Bürgmann, P. A. Rosen, and E. J. Fielding, "Synthetic aperture radar interferometry to measure earth's surface topography and its deformation," Annual review of earth and planetary sciences, vol. 28, no. 1, pp. 169-209, 2000.

[5] J. Elliott, R. Walters, and T. Wright, "The role of space-based observation in understanding and responding to active tectonics and earthquakes," Nature communications, vol. 7, p. 13844, 2016.

[6] R. F. Hanssen, Radar interferometry: data interpretation and error analysis. Springer Science \& Business Media, 2001, vol. 2.

[7] T. Emardson, M. Simons, and F. Webb, "Neutral atmospheric delay in interferometric synthetic aperture radar applications: Statistical description and mitigation," Journal of Geophysical Research: Solid Earth, vol. 108, no. B5, 2003. 
[8] R. B. Lohman and M. Simons, "Some thoughts on the use of insar data to constrain models of surface deformation: Noise structure and data downsampling," Geochemistry, Geophysics, Geosystems, vol. 6, no. 1, 2005.

[9] F. Onn and H. Zebker, "Correction for interferometric synthetic aperture radar atmospheric phase artifacts using time series of zenith wet delay observations from a gps network," Journal of Geophysical Research: Solid Earth, vol. 111, no. B9, 2006.

[10] H. A. Zebker and J. Villasenor, "Decorrelation in interferometric radar echoes," IEEE Transactions on geoscience and remote sensing, vol. 30, no. 5, pp. 950-959, 1992.

[11] D. Just and R. Bamler, "Phase statistics of interferograms with applications to synthetic aperture radar," Applied optics, vol. 33, no. 20, pp. 4361-4368, 1994.

[12] F. De Zan, A. Parizzi, P. Prats-Iraola, and P. López-Dekker, "A sar interferometric model for soil moisture," IEEE Transactions on Geoscience and Remote Sensing, vol. 52, no. 1, pp. 418-425, 2013.

[13] F. De Zan, M. Zonno, and P. López-Dekker, "Phase inconsistencies and multiple scattering in sar interferometry," IEEE Transactions on Geoscience and Remote Sensing, vol. 53, no. 12, pp. 6608-6616, 2015.

[14] S. Zwieback, X. Liu, S. Antonova, B. Heim, A. Bartsch, J. Boike, and I. Hajnsek, "A statistical test of phase closure to detect influences on dinsar deformation estimates besides displacements and decorrelation noise: Two case studies in high-latitude regions," IEEE Transactions on Geoscience and Remote Sensing, vol. 54, no. 9, pp. 5588-5601, 2016.

[15] S. Zwieback and F. J. Meyer, "Repeat-pass interferometric speckle," IEEE Transactions on Geoscience and Remote Sensing, pp. 1-15, 2020.

[16] H. Ansari, F. De Zan, and A. Parizzi, "Study of systematic bias in measuring surface deformation with sar interferometry," IEEE Transactions on Geoscience and Remote Sensing, pp. 1-1, 2020.

[17] A. Ferretti, C. Prati, and F. Rocca, "Permanent scatterers in sar interferometry," IEEE Transactions on geoscience and remote sensing, vol. 39, no. 1, pp. 8-20, 2001.

[18] A. Hooper, H. Zebker, P. Segall, and B. Kampes, "A new method for measuring deformation on volcanoes and other natural terrains using insar persistent scatterers," Geophysical research letters, vol. 31, no. 23, 2004.

[19] P. Berardino, G. Fornaro, R. Lanari, and E. Sansosti, "A new algorithm for surface deformation monitoring based on small baseline differential sar interferograms," IEEE transactions on geoscience and remote sensing, vol. 40, no. 11, pp. 2375-2383, 2002.

[20] A. M. Guarnieri and S. Tebaldini, "Hybrid cramér-rao bounds for crustal displacement field estimators in sar interferometry," IEEE signal processing letters, vol. 14, no. 12, pp. 1012-1015, 2007.

[21] A. M. Guarnieri and S. Tebaldini, "On the exploitation of target statistics for sar interferometry applications," IEEE Transactions on Geoscience and Remote Sensing, vol. 46, no. 11, pp. 34363443, 2008.

[22] A. Ferretti, A. Fumagalli, F. Novali, C. Prati, F. Rocca, and A. Rucci, "A new algorithm for processing interferometric data-stacks: Squeesar," IEEE Transactions on Geoscience and Remote Sensing, vol. 49, no. 9, pp. 3460-3470, 2011.

[23] G. Fornaro, S. Verde, D. Reale, and A. Pauciullo, "Caesar: An approach based on covariance matrix decomposition to improve multibaseline-multitemporal interferometric sar processing," IEEE Transactions on Geoscience and Remote Sensing, vol. 53, no. 4, pp. 2050-2065, 2014.

[24] H. Ansari, F. De Zan, and R. Bamler, "Sequential estimator: Toward efficient insar time series analysis," IEEE Transactions on Geoscience and Remote Sensing, vol. 55, no. 10, pp. 5637$5652,2017$.

[25] H. Ansari, F. De Zan, and R. Bamler, "Efficient phase estimation for interferogram stacks," IEEE Transactions on Geoscience and Remote Sensing, vol. 56, no. 7, pp. 4109-4125, 2018.

[26] F. Rocca, "Modeling interferogram stacks," IEEE Transactions on Geoscience and Remote Sensing, vol. 45, no. 10, pp. 32893299, 2007

[27] P. Agram and M. Simons, "A noise model for insar time series," Journal of Geophysical Research: Solid Earth, vol. 120, no. 4, pp. 2752-2771, 2015.

[28] S. Samiei-Esfahany and R. Hanssen, "On the evaluation of second order phase statistics in sar interferogram stacks," Earth Observation and Geomatics Engineering, vol. 1, no. 1, pp. 115, 2017.

[29] B. Barber, "The phase statistics of a multichannel radar interferometer," Waves in random media, vol. 3, no. 4, pp. 257-266, 1993.

[30] J.-S. Lee, K. W. Hoppel, S. A. Mango, and A. R. Miller, "Intensity and phase statistics of multilook polarimetric and interferometric sar imagery," IEEE Transactions on Geoscience and Remote Sensing, vol. 32, no. 5, pp. 1017-1028, 1994.

[31] L. Joughin and D. Winebrenner, "Effective number of looks for a multilook interferometric phase distribution," in Proceedings of IGARSS'94-1994 IEEE International Geoscience and Remote Sensing Symposium, vol. 4. IEEE, 1994, pp. 2276-2278.

[32] J. Tough, D. Blacknell, and S. Quegan, "A statistical description of polarimetric and interferometric synthetic aperture radar data," Proceedings of the Royal Society of London. Series A: Mathematical and Physical Sciences, vol. 449, no. 1937, pp. 567-589, 1995.

[33] E. Rodriguez and J. Martin, "Theory and design of interferometric synthetic aperture radars," IEE Proceedings F (Radar and Signal Processing), vol. 139, no. 2, pp. 147-159, 1992.

[34] A. Parizzi, X. Cong, and M. Eineder, "First results from multifrequency interferometry. a comparison of different decorrelation time constants at $1, \mathrm{c}$, and $\mathrm{x}$ band," ESA Scientific Publications, no. SP-677, pp. 1-5, 2009.

[35] Y. Morishita and R. F. Hanssen, "Temporal decorrelation in 1-, c-, and x-band satellite radar interferometry for pasture on drained peat soils," IEEE Transactions on Geoscience and Remote Sensing, vol. 53, no. 2, pp. 1096-1104, 2014.

[36] H. A. Zebker, P. A. Rosen, and S. Hensley, "Atmospheric effects in interferometric synthetic aperture radar surface deformation and topographic maps," Journal of geophysical research: solid earth, vol. 102, no. B4, pp. 7547-7563, 1997.

[37] T. Wright, B. Parsons, and E. Fielding, "Measurement of interseismic strain accumulation across the north anatolian fault by satellite radar interferometry," Geophysical Research Letters, vol. 28, no. 10, pp. 2117-2120, 2001.

[38] T. J. Wright, B. Parsons, P. C. England, and E. J. Fielding, "Insar observations of low slip rates on the major faults of western tibet," Science, vol. 305, no. 5681, pp. 236-239, 2004.

[39] O. Cavalié, C. Lasserre, M.-P. Doin, G. Peltzer, J. Sun, X. Xu, and Z.-K. Shen, "Measurement of interseismic strain across the haiyuan fault (gansu, china), by insar," Earth and Planetary Science Letters, vol. 275, no. 3-4, pp. 246-257, 2008.

[40] D. Schmidt, R. Bürgmann, R. Nadeau, and M. d'Alessio, "Distribution of aseismic slip rate on the hayward fault inferred from seismic and geodetic data," Journal of Geophysical Research: Solid Earth, vol. 110, no. B8, 2005.

[41] M. Furuya and S. Satyabala, "Slow earthquake in afghanistan detected by insar," Geophysical Research Letters, vol. 35, no. 6, 2008 . 
[42] H. Dragert, K. Wang, and T. S. James, "A silent slip event on the deeper cascadia subduction interface," Science, vol. 292, no. 5521, pp. 1525-1528, 2001.

[43] M. M. Miller, T. Melbourne, D. J. Johnson, and W. Q. Sumner, "Periodic slow earthquakes from the cascadia subduction zone," Science, vol. 295, no. 5564, pp. 2423-2423, 2002.

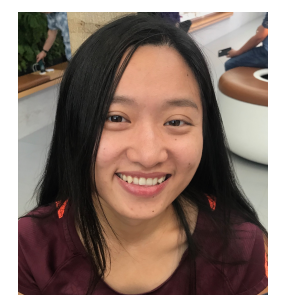

Yujie Zheng received the B.S. degree from Peking University, Beijing, China, in 2014, and the Ph.D. degree in Geophysics from Stanford University, CA, USA, in 2019. She is currently a Postdoctoral Scholar in the Seismological Laboratory, California Institute of Technology. Her main research interests are Interferometric synthetic aperture radar (InSAR) processing and InSAR applications in crustal deformation studies - earthquakes, volcanoes, anthropogenic signals of deformation such as water management or oil and gas resources.

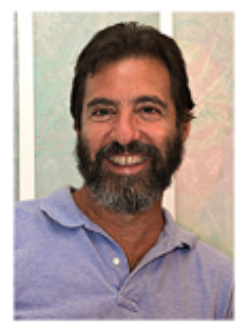

Howard Zebker (M'87-SM'89-F'99) received the B.S. degree from the California Institute of Technology, Pasadena, CA, USA, in 1976, the M.S. degree from the University of California at Los Angeles, Los Angeles, CA, USA, in 1979, and the Ph.D. degree in electrical engineering from Stanford University, Stanford, CA, USA, in 1984. He is currently a Professor of geophysics and electrical engineering with Stanford University, Stanford, CA, USA, where his research group specializes in interferometric radar remote sensing. He was a Microwave Engineer with the NASA Jet Propulsion Laboratory (JPL), Pasadena, where he built support equipment for the SEASAT satellite synthetic aperture radar and designed airborne radar systems. He later developed imaging radar polarimetry, a technique for measurement of the radar scattering matrix of a surface. He is best known for the development of radar interferometry, leading to spaceborne and airborne sensors capable of measuring topography to meter scale accuracy and surface deformation to millimeter scale. More recently, he has been participating in the NASA Cassini Mission to Saturn, concentrating on analysis of data acquired by the radar/radiometer instrument.

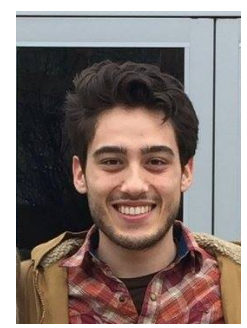

Roger Michaelides received the B.A. degree in physics and science of earth systems from Cornell University, Ithaca, NY, USA, in 2015, the Ph.D. degree in geophysics from Stanford University, Stanford, CA, USA in 2020. He is currently a Postdoctoral Researcher in the Department of Geophysics at the Colorado School of Mines. His research interests include radar remote sensing applications for terrestrial and planetary geophysics, especially InSAR algorithm development, quantifying uncertainties in InSAR retrieval algorithms, and InSAR applications for studying hydrologic, cryologic, environmental processes in periglacial and vegetated landscapes, radar remote sensing for planetary science applications, and altimtetry and imaging radar instrument onboard the Cassini mission to Saturn. 